\title{
Endoscopic treatment of Zenker's diverticulum using a new triangle tip knife
}

\author{
Zaheer Nabi, Mohan Ramchandani, Nitin Jagtap, Santosh Darisetty, D. Nageshwar Reddy \\ Asian Institute of Gastroenterology, Hyderabad, India
}

\begin{abstract}
Background Zenker's diverticulum (ZD) is a rare disease that affects elderly subjects. The mainstay of management is endoscopic myotomy of the cricopharyngeal septum. In this series, we aimed to evaluate the feasibility and efficacy of cricopharyngeal myotomy with a new electrosurgical knife in patients with ZD.

Methods Consecutive patients with ZD who underwent flexible endoscopic myotomy with the new triangle tip knife J (TTJ) were included in the study. The study outcomes included technical and clinical success, procedure duration, and adverse events.

Results A total of 7 patients [median age 57, (range 40-87) years] underwent cricopharyngeal myotomy with the TTJ knife. The median size of the ZD was 4 (range $3-5) \mathrm{cm}$. The procedure was successfully completed in all the patients with a median operative time of 12 (range 8-16) min. There were no major adverse events. Minor bleeding was noticed in 3 patients and was successfully managed using the same knife. Clinical resolution of symptoms was noticed in all the patients at follow up.
\end{abstract}

Conclusion Cricopharyngeal myotomy can be performed safely and efficiently with the new TTJ knife.

Keywords Endoscopy, diverticulum, esophagus, myotomy

Ann Gastroenterol 2019; 32 (5): 1-4

\section{Introduction}

Zenker's diverticulum (ZD) is a posterior pharyngoesophageal outpouching caused by the dysfunction of cricopharyngeal muscle [1]. ZD is rare and predominantly effects elderly males during the sixth to eighth decades of life. The predominant symptoms include dysphagia, regurgitation of undigested food, aspiration, cough, and nutritional compromise.

The mainstay of treatment for $\mathrm{ZD}$ is cricopharyngeal myotomy, performed either surgically or by endoscopic methods. Over the last few decades, the management of ZD has shifted from surgery to transoral flexible endoscopic myotomy.

Gastroenterology Department, Asian Institute of Gastroenterology, Hyderabad, India

Conflict of Interest: None

Correspondence to: Zaheer Nabi, Consultant Gastroenterologist, Asian Institute of Gastroenterology, 6-3-661, Somajiguda, Hyderabad, 500 082, India, e-mail: drzaheeraig@gmail.com

Received 5 April 2019; accepted 11 May 2019; published online 17 June 2019

DOI: https://doi.org/10.20524/aog.2019.0393
Compared to surgery, endoscopic treatment results in a shorter procedure and hospitalization, earlier diet introduction, and lower complication rate [2]. Flexible endoscopic myotomy involves division of the cricopharyngeal septum using electrosurgical knives. There is no dedicated knife designed specifically for the endoscopic treatment of ZD. Consequently, the use of various knives has been described in the literature, with variable outcomes.

In this case series, we report our experience on patients with ZD who underwent cricopharyngeal myotomy using a novel triangle tip knife J (TTJ).

\section{Patients and methods}

The study was conducted at a tertiary care center. All patients with ZD who underwent flexible endoscopic cricopharyngeal myotomy using the TTJ (Fig. 1) were enrolled in the study.

\section{Procedure technique}

All the procedures were performed under general anesthesia with the patient in the left lateral position in an endoscopy suite. 
Prior to the start of the procedure, an endoscopy was performed under light sedation to clear the contents of the diverticulum. Subsequently, a nasogastric tube was placed over the wire. A standard adult upper gastrointestinal endoscope (GIF Q 190, Olympus, Tokyo, Japan) with a distal attachment ( $\mathrm{MH}-593$, Olympus, Tokyo, Japan) was used for the procedure. The use of a diverticuloscope was left to the discretion of the operator. Cricopharyngeal myotomy was performed with a TTJ knife.

The steps of the myotomy were as follows (Fig. 2A-F). Initially, the mucosa and submucosa were cut using the TTJ knife in endocut mode (Endo cut Q, effect 1) to expose the muscle fibers (Fig. 2A,B). Subsequently, the muscle fibers were divided using the same knife in spray coagulation (40 W, effect 2) mode. The muscle fibers were divided in an incremental manner as far as the lower end of diverticulum. Minor intraprocedural bleeding was controlled with the TTJ knife in spray coagulation mode (Fig. 2C,D). Brisk or pulsatile bleeding was controlled using coagulation forceps and soft coagulation mode ( $80 \mathrm{~W}$, effect 5 ). The depth of the remaining part of the diverticulum was assessed intermittently using the

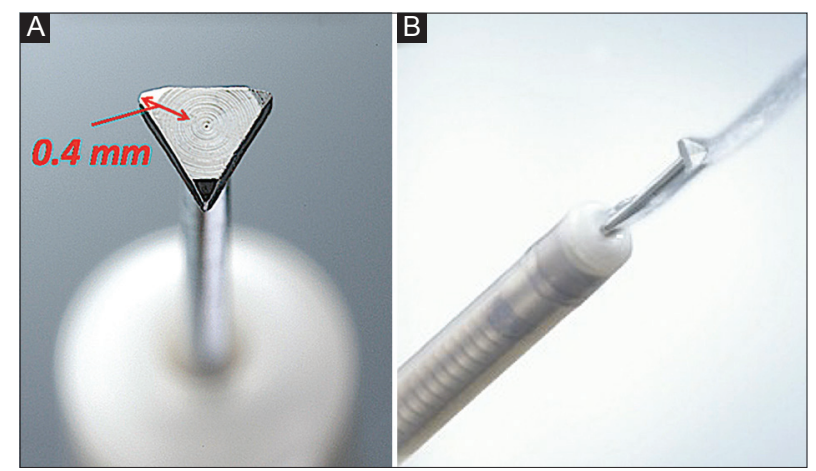

Figure 1 Triangle tip knife J (A) The triangle tip knife has a length of $0.4 \mathrm{~mm}$; (B) Note the integrated water jet facility catheter of the knife to guide the distal extent of myotomy (Fig. 2E). Towards the lower end of the diverticulum, the knife's triangle tip was used to precisely hook and dissect the muscle fibers of the bridge. After the division of the cricopharyngeal septum was completed, a prophylactic clip was applied at the bottom of the myotomy site (see Video, Supplemental Digital Content 1, which demonstrates the technique of endoscopic diverticulotomy) (Fig. 2F).

\section{Post-procedure follow up}

A follow-up barium swallow was performed 24-48 h after the procedure, following which oral diet was resumed. Subsequently, the patients were followed-up at 3 months, 1 year, and yearly thereafter.

\section{Outcome measures}

Technical and clinical success were recorded. In addition, the time required for the completion of the procedure was documented, as well as any intraprocedural and immediate postoperative adverse events. Operative time was defined as time taken from the initiation of the mucosectomy to the application of endoclips. Time spent placing the nasogastric tube and positioning the diverticuloscope was not included in the procedure duration.

\section{Devices and accessories}

A new electrosurgical knife was used for cricopharyngeal myotomy: the TTJ knife (Triangle Tip Knife J, KD-645L, Olympus, Japan). We have previously described the use of
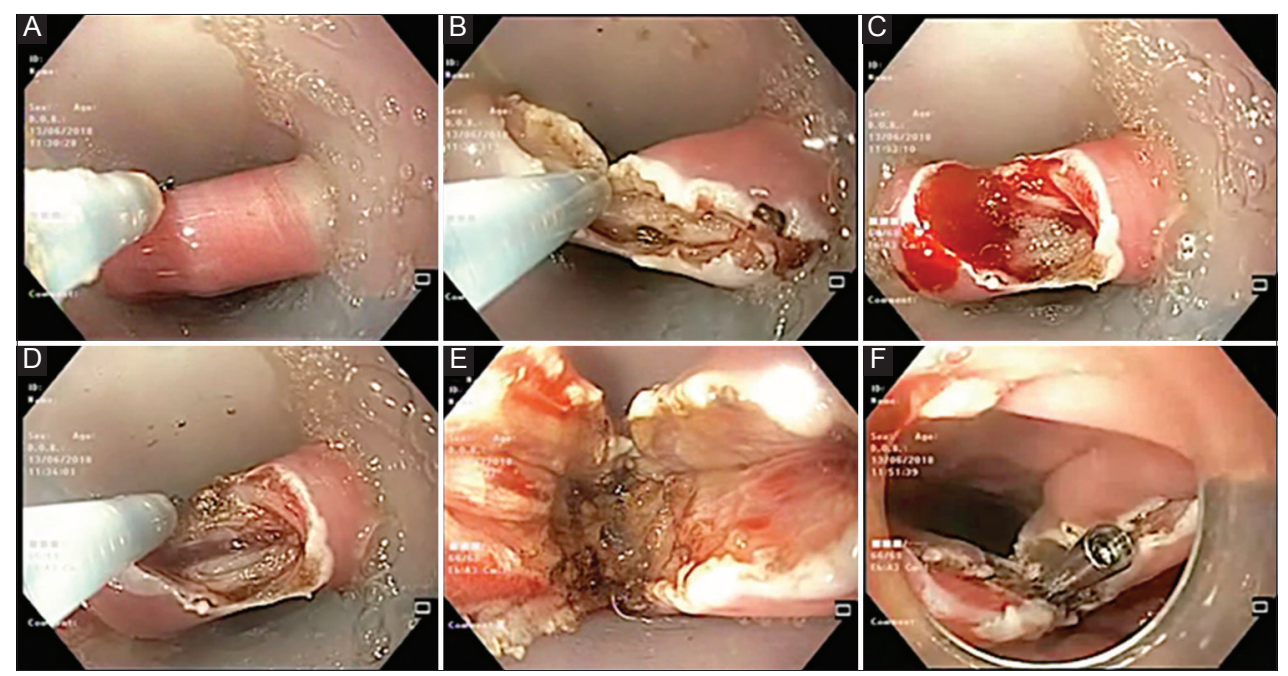

Figure 2 Endoscopic diverticulotomy using new triangle tip knife (A) Endoscopic view of Zenker's diverticulum with soft diverticuloscope in situ separating the lips of cricopharyngeal septum; (B) Myotomy using new triangle tip knife with integrated water jet system; (C) Minor ooze at the site of dissection; (D) Control of bleeding with spray coagulation using the same knife; (E) Endoscopic view after the completion of diverticulotomy; (F) Application of endoclips at the base of diverticuluotomy site 
this knife for peroral endoscopic myotomy [3]. The TTJ knife has a triangle tip with a length and thickness of $0.4 \mathrm{~mm}$ and $0.3 \mathrm{~mm}$, respectively. The cutting knife length and diameter are $4.5 \times 0.4 \mathrm{~mm}$. The TTJ knife is equipped with a water jet nozzle (Fig. 1A,B).

Other accessories included an electrosurgical unit (VIO300D; ERBE, Tübingen, Germany), a soft plastic diverticuloscope with double duckbill end (ZDO-22-30; Cook Endoscopy, Winston-Salem, North Carolina), coagulation forceps (Coagrasper G, FD-412LR, Olympus), and endoscopic clips (EZ Clip, HX-610-090L; Olympus Corp). The settings on the electrosurgical unit with this knife were ENDO CUT Q at $40 \mathrm{~W}$, effect 2 for spray coagulation. Soft coagulation mode (W 80, effect 5) was used for brisk bleeding.

\section{Results}

A total of 7 patients [median age 57 (range $40-87$ ) years, $80 \%$ male] underwent cricopharyngeal myotomy for symptomatic ZD with TTJ knife. A soft diverticuloscope was used in 3 patients. The median size of the diverticulum was 4 (range $3-5$ ) $\mathrm{cm}$, and the median duration of symptoms was 10 months. Median procedure time was 12 (range 8-16) min. Three patients had a mild intraprocedural bleed managed endoscopically with the TTJ knife itself. On oral contrast study, a free flow into the esophagus was documented in all patients. One patient (size of ZD $5 \mathrm{~cm}$ ) underwent a planned second session of cricopharyngeal myotomy at an interval of 4 weeks. All 7 patients were asymptomatic at 3 months of follow up. Five patients completed 1-year follow up and had no recurrence of symptoms (Table 1).

\section{Discussion}

In this case series, we described the use of a new knife for the treatment of ZD. Cricopharyngeal myotomy with the new TTJ knife was found to be safe, effective and rapid. The management of $\mathrm{ZD}$ has evolved from surgery to flexible endoscopic cricopharyngeal myotomy. The aim of flexible endoscopic treatment is complete division of the cricopharyngeal septum, which divides the lumen of the diverticulum and esophagus. Use of different knives has been described for cricopharyngeal myotomy, including the needle knife, hook knife, stag-beetle knife, and clutch cutter device [4-7]. However, none of the aforementioned knives was specifically designed for this purpose. With recent advancements in third-space endoscopy, new electrosurgical knives are available that have been designed for the effective division of muscle fibers. In the current series, we described the use of one such knife, a triangle tip knife with a water jet. The authors had previous experience with the TTJ knife for peroral endoscopic myotomy $[3,8]$.

The cricopharyngeal septotomy procedure was successfully completed in all 7 patients and there were no major adverse events. The use of the TTJ knife was found to be technically

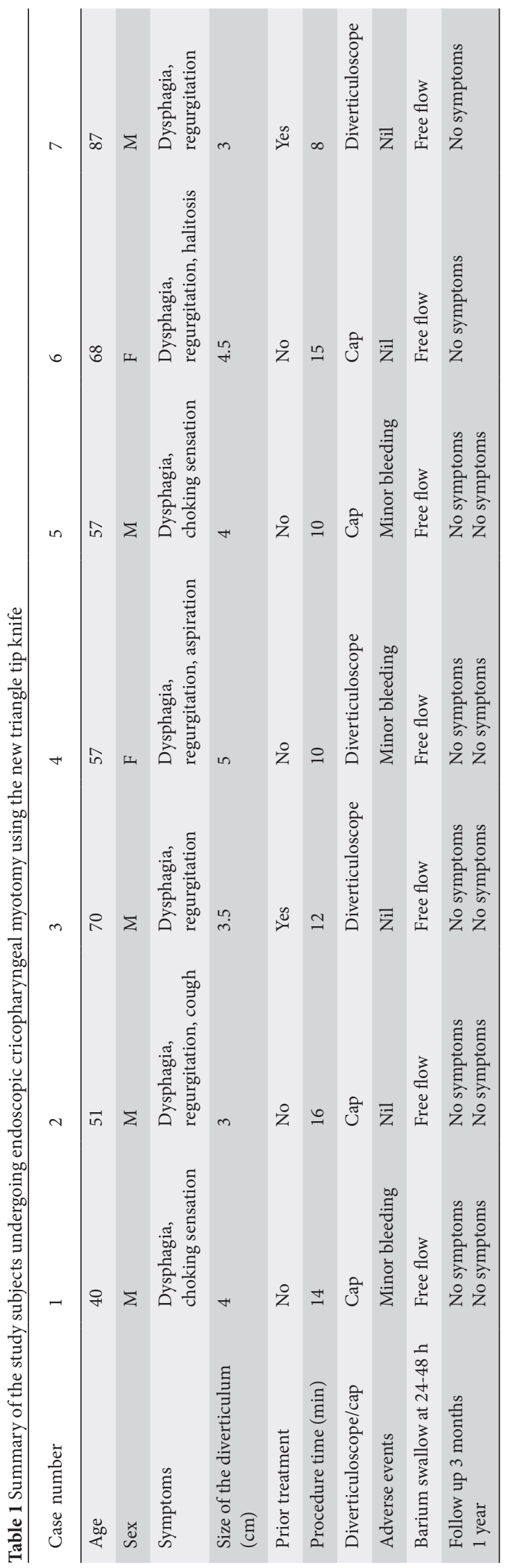


easy and there was no need for additional accessories in any of the cases. The median procedure time in the present series was only $12 \mathrm{~min}$. The TTJ knife has a distal L-shaped hook in three directions, which obviates the need to rotate the knife. In addition, the spray coagulation mode allows efficient and quick myotomy. Both these features of the TTJ knife allow fast completion of the procedure. In one patient the diverticulotomy was carried out in 2 sessions, given our relative inexperience with the new knife for $\mathrm{ZD}$ and the large size of the diverticulum in that case.

There were no major or minor adverse events during the procedure. Minor bleeding was documented in 3 patients and could be easily controlled with the triangle tip knife itself. Intraprocedural bleeding is not uncommon during cricopharyngeal myotomy and often requires an additional accessory, such as coagulation forceps, to achieve hemostasis [9]. A distinct advantage of the TTJ knife over other knives is the precise localization of the site of bleeding, as it has an integrated water jet function. In addition, spray coagulation mode provides effective coagulation without the need to actually contact the bleeding site. Therefore, the need for extra equipment such as coagulation forceps is avoided and operative time is reduced as well as procedural cost.

Besides bleeding, the other and more feared complication of cricopharyngeal myotomy is perforation [9]. Overzealous dissection towards the lower end of the septum may result in perforation. On the other hand, incomplete division of fibers may lead to recurrence of symptoms [10]. Therefore, the division of muscle fibers at the lower end of septum should be precise and meticulous. In this regard, TTJ knife may be potentially advantageous owing to its ability to hook small portions of muscle fibers for accurate dissection at the lower end of the septum. The sharp triangle hooks are oriented in three directions, obviating the need to rotate the knife to catch the fibers.

In the present series, several additional factors may have influenced the safety and efficacy of endoscopic diverticulotomy. These include use of a nasogastric tube and diverticuloscope (some cases), placement of a prophylactic clip at the bottom of the myotomy, and the previous experience of the operators in performing endoscopic myotomies.
In conclusion, cricopharyngeal myotomy can be safely and efficiently performed using the new TTJ knife. The integrated water jet, efficient coagulation and capability to precisely deliver spray coagulation current with this knife allow the procedure to be accomplished quickly, without the need of additional accessories. Larger comparative trials are required to compare different knives and techniques for flexible endoscopic myotomy in ZD.

\section{References}

1. Law R, Katzka DA, Baron TH. Zenker's diverticulum. Clin Gastroenterol Hepatol 2014;12:1773-1782.

2. Albers DV, Kondo A, Bernardo WM, et al. Endoscopic versus surgical approach in the treatment of Zenker's diverticulum: systematic review and meta-analysis. Endosc Int Open 2016;4:E678-E686.

3. Nabi Z, Ramchandani M, Chavan R, Kalapala R, Darisetty S, Reddy DN. Outcome of peroral endoscopic myotomy in achalasia cardia: experience with a new triangular knife. Saudi J Gastroenterol 2018;24:18-24.

4. Vogelsang A, Preiss C, Neuhaus H, Schumacher B. Endotherapy of Zenker's diverticulum using the needle-knife technique: long-term follow-up. Endoscopy 2007;39:131-136.

5. Repici A, Pagano N, Romeo F, et al. Endoscopic flexible treatment of Zenker's diverticulum: a modification of the needle-knife technique. Endoscopy 2010;42:532-535.

6. Ramchandani M, Nageshwar Reddy D. New endoscopic "scissors" to treat Zenker's diverticulum (with video). Gastrointest Endosc 2013;78:645-648.

7. Neumann H, Löffler S, Rieger S, Kretschmer C, Nägel A. Endoscopic therapy of Zenker's diverticulum using a novel endoscopic scissor - the Clutch Cutter device. Endoscopy 2015;47 Suppl 1 UCTN: E430-E431.

8. Nabi Z, Ramchandani M, Chavan R, et al. Peroral endoscopic myotomy in children: first experience with a new triangular knife. J Pediatr Gastroenterol Nutr 2018;66:43-47.

9. Li LY, Yang YT, Qu CM, et al. Endoscopic needle-knife treatment for symptomatic esophageal Zenker's diverticulum: A metaanalysis and systematic review. J Dig Dis 2018;19:204-214.

10. Costamagna G, Iacopini F, Bizzotto A, et al. Prognostic variables for the clinical success of flexible endoscopic septotomy of Zenker's diverticulum. Gastrointest Endosc 2016;83:765-773. 